\title{
Outbreak of Neonatal Septicaemia at a Tertiary care Hospital due to Enterobacter cloacae
}

\author{
Dr.KaranIndra Ostwal ${ }^{1 *}$, Dr.TruptiAmol Joshi ${ }^{1 \#,}$ \\ Dr.PoonamAshokraoKadam ${ }^{2 *}$ Dr.ReenaRamchandra Karkhele ${ }^{2 \#}$ Dr.Jyoti Anil \\ Iravane $^{3 *}$, Dr.LaxmanikantSeshrao Deshmukh ${ }^{3 \#,}$ Dr.AjitShreeram Damle ${ }^{4 *}$, \\ *Department of Microbiology \# Department of Neonatology \\ ${ }^{1}$ Assistant Professor,Department of Microbiology, Dr.VMGMCSolapur (MS) INDIA \\ ${ }^{1}$ Assistant Professor, Department of Paediatrics GMC Aurangabad (MS) INDIA \\ ${ }^{2,2}$ Junior Resident, Department of Microbiology \& Paediatrics GMC Aurangabad (MS) INDIA \\ ${ }^{3}$ Associate Professor, Department of Microbiology, GMC Aurangabad (MS)INDIA \\ ${ }^{3}$ Professor \& Head of Neonatology, Department of Paediatrics GMC Aurangabad (MS) INDIA \\ ${ }^{4}$ Professor \& Head of Department, Microbiology GMC Aurangabad (MS)INDIA
}

\begin{abstract}
:
Introduction:Neonatal septicaemia is a haunting problem for neonatologists all over the world with considerable morbidity and mortality. In the Indian scenario, members of Enterobacteriaceae continue to be a challenging problem for the sick and debilitated neonates.

Materials And Methods:Outbreak was notified by increased isolation of E. cloacae from blood culture. Investigation of outbreak was done by taking handprints, glove juice culture, rectal swab culture of neonates \& by environmental sampling.

Results:There were 40\%(26/65) E. cloacae isolated from blood culture of suspected septicemic neonates in Dec.2013 compared to 7\%(5/68) in Nov.2013 \& 8\%(5/56) in Jan.2014; clearly indicating outbreak in Dec.2013. During outbreak investigation we could isolate E. cloacae from handprints of two staff nurse, from glove juices of four relatives \& from rectal swabs of four neonates. There was no isolation of E. cloacae after extensive environmental sampling.

Conclusions: Clinical significance of E. cloacae as a neonatal pathogen warrants the attention of clinicians as well as microbiologists. Breaches in infection control measures should be avoided to prevent such outbreaks.
\end{abstract}

Keywords: Enterobacter cloacae, Health care associated Infection, Neonatal Septicaemia

\section{Introduction}

Neonatal septicaemia (NS) is a haunting problem for neonatologists all over the world. This clinical entity is more commonly associated with Preterm and low birthweightbabies ${ }^{(1)}$. Neonatal septicaemia is difficult to diagnose clinically as it presents with non-specific and usually subtle signs and symptoms ${ }^{(2)}$.In the Indian scenario,members of familyEnterobacteriaceae continue to be a challenging problem for the sick and debilitated neonates. The recent literature review has revealed the significant role of speciesof the genus Enterobacter such as Enterobacter cloacae, Enterobacter sakazaki, Enterobacteraerogenes in causing infections, especially in neonatal intensive care ${ }^{(1)}$. Health care-associated infections occurring in NeonatalIntensive Care Unit (NICU) is likely to have disastrous consequences for affected patients and raise enormous concerns in staff. Only a small proportion of these outbreaks have been published in the medical literature, hence representing the tip of the iceberg only. However, this information is extremely valuable for gaining a better insight into the nature of the development of neonataloutbreaks and to improve outbreak investigation and prevention ${ }^{(3)}$.

\section{Material \& Methods}

In the present study, a case of late onset neonatal sepsis was defined as an infant who had clinical signs of infection or those who were born to mothers with risk factors for infection, in whom blood culture obtained after 72 hours of life, grew a bacterial pathogen. Clinical signs of infection included poor feeding, lethargy, respiratory problems (such as tachypnea, apnea, grunting, cyanosis, and retraction), temperature instability (such as hyperthermia ), gastrointestinal problems (as vomiting, abdominal distension, diarrhoea), and central nervous system symptoms (such as convulsion, hypotonia, and irritability) ${ }^{(4)}$. The identification of the strain was done by conventional biochemical tests(Fig-1). The strain typing was done based on Antibiogram. The antibiotic susceptibility testing was done by Kirby-Bauer disc diffusion test. ESBL production by the strain was phenotypically confirmed by combined disc test ${ }^{(5)}$.Investigation of the outbreak was carried out first by carrying out environmental sampling. Swabs were taken both from labour room and NICU. Total of 24 samples were 
collected from NICU including linen, humidifier, incubator, cradle, refrigerator, feeding trolley, tap water, suction fluid, umbilical cord stump of neonates,temperature sensors, suction tips. Ten samples were collected from labour room including linen, tap water, weighing machine, curtains and cradles. All the 34 environmental samples were collected in trypticase soy Broth (TSB) and sub-cultured after overnight incubation on MacConkey agar and Blood agar ${ }^{(6)}$.

Screening of Health care workers (HCWs) - Rounds were carried out in NICU and cross sectionally the hand prints were taken of the HCWs present at that time including the doctors, nurses and ward boys in all twelve persons were screened.

Screening of Babies relatives - screening of relatives was also done by hand Print and glove juice method. Both the relatives of septic babies(clinically suspected) and aseptic babies were involved in the screening. Four relatives of septic babies and four of aseptic babies taken as control were taken by both hand print and glove juice method $^{(6)}$.

Rectal screening of the babies - In clinically suspected and laboratory proven cases of septic babies' rectal swabs were taken to look for the causative organism. Rectal swabs were taken from four of clinically suspected septic babies and four from aseptic babies (taken as control) for culture and sensitivity

Technique for glove juice method - For that powder free hand-gloves were given to the relative of the babies to wear then $25 \mathrm{ml}$ of nutrient broth was introduced aseptically from the side of the glove worn by mothers. After massaging the hands with gloves for one minute the broth was taken out by syringe with needle \& cultured on agar plates.

\section{Results}

There were $40 \%(26 / 65)$ E. cloacae isolated from blood culture of suspected septicaemia neonates in December 2013 compared to 7\%(5/68) in Nov.2013 \&8\%(5/56) in Jan.2014; clearly indicating outbreak in Dec.2013. All the strains of E.cloacae were found to be Extended Spectrum Beta Lactamase (ESBL) producers with susceptibility only to Cefoperazone-sulbactam and Carbapenem class drugs ${ }^{(7)}$. In environmental sampling of labour room and NICU no source of Enterobacter cloacae could be ascertained. The screening of HCWs showed pure growth of Enterobacter cloacaein two staff nurses with hand print method. The screening of the relatives showed all the relatives attending the babies who were culture positive(Enterobacter cloacae) cases of sepsis had pure growth of Enterobacter cloacae on their hands. We also evaluated the efficacy of the hand print and glove juice technique in determining the bacterial load on the hands of the relatives attending the babies.

Table-1 Comparison of techniques used for screening

\begin{tabular}{|l|l|l|}
\hline Method used $(\mathrm{n}=8)$ & $\begin{array}{l}\text { Relative of Septic Baby } \\
(\mathrm{n}=4)\end{array}$ & $\begin{array}{l}\text { Relative of Aseptic Baby } \\
(\mathrm{n}=4)\end{array}$ \\
\hline 1.Hand Print & 2 & 0 \\
\hline 2. Glove Juice & 4 & 0 \\
\hline
\end{tabular}

Thus the glove juice technique was found to be very effective in screening of the cases.

The rectal screening performed in all the septic babies (four) had pure growth of Enterobacter cloacae.Aseptic babies did not have Enterobacter cloacaein rectal swab cultures

Identification of Reservoir -After noting the above findings relatives and the staff nurses who had Enterobacter cloacae on hands were interviewed in detail. A common thing was found that was the use of a particular water closet. Accordingly samples were taken from the water closet door handles and tap to find the same kind of strain on both the sites.

\section{Corrective actions taken -}

1.The golden rule of through Hand washing was re-emphasized

2. Restriction to the entry of relative/mother was made more stringent

3.A protocol for house -keeping and environmental cleaning was made and ensured to be followed by everyone.

4. The water closet harbouring Enterobacter cloacae was thoroughly disinfected.

\section{Discussion-}

Enterobacter species are frequent components of NICU flora and may now be more common than Klebsiella as causes of neonatal infections. Outbreaks have been associated with contaminated infant formula and intravenous fluids ${ }^{(8)}$. Several hospital outbreaks traced to contaminated parenteral fluid solutions have implicated Enterobacterspecies. In addition to ampicillin, most isolates are resistant to first-generation cephalosporins, but may be susceptible to second- or third-generation cephalosporins; however, mutants 
derepressed for $\beta$-lactamase production occur at relatively high frequency and confer resistance to many

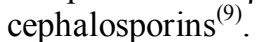

Sepsis is still a major cause of mortality and morbidity in the first month of life. New treatment alternatives are being explored throughout the world because of its changing bacterial profile and high mortality rate. Gram-negative organisms continue to be a menace to the sick, fragile and debilitated newborns ${ }^{(10)}$. In the present study Enterobacter cloacae has been isolated as the cause of sepsis

similar findings have been noted by Beena et $\mathrm{al}^{(1)}$ Rastogi et al ${ }^{(2)}$ and Mahapatra et $\mathrm{al}^{(10)}$. In our NICU there was compulsion for hand washing \& wearing gowns while entering into unit, but there was breach in continuity of infection control leading to outbreak. To investigate the cross contamination in NICU we took handprints of all health care workers in the unit \& we got Enterobacter cloacae on the hands of a staff nurse. So we believe that relatives attending the baby \& staff nurse were responsible for the outbreak in NICU. The strain of Enterobacter cloacaeisolated from all blood cultures i.efrom relative's hand,hands of staff nurse and water closet showed same Antibiogram.Overcrowding and understaffing i.e low nurse to patient ratio may be a factor for the breach in aseptic protocols leading to outbreaks ${ }^{(11,12)}$. The baby's skin, respiratory tract, conjunctivae, gastrointestinal (GI) tract, and umbilicus may become colonized from the environment and such colonization lead to the possibility of late-onset sepsis from invasive microorganisms. Vehicle for such colonization may include vascular or urinary catheters, other indwelling lines, or contact with caregivers who have bacterial colonization ${ }^{(12)}$. Bloodstream infections are not only the most frequent health care-associated infections in NICU outbreaks; they also represent the most frequent endemic infections in neonates. Extensive use of indwelling catheters and prolonged parenteral nutrition in sick infants are some of the reasons for the high prevalence of bloodstream infections ${ }^{(13,14)}$. Infection control staff should always aim for the most effective way of outbreak investigations to stop pathogen spread as soon as possible.

Any suspicion of an outbreak should lead to a review of general infection control procedures, with the emphasis on compliance with hand disinfection betweenall contacts with patients. Furthermore, practices for sterilization and disinfection of equipment, the preparation of infant formulae, and aseptic techniques for invasive procedures should be reviewed ${ }^{(15,16)}$.

Enterobacter cloacae septicaemia should be emphasised and taken into account for its rising incidence. Moreover Enterobacter cloacaeaffects the most vulnerable age group i.e. neonates and its growing resistance to conventional and even newer antibiotics is a serious cause for concern. Thus, its containment and prevention needs special consideration.

\section{Conclusions}

Clinical significance of Enterobacter cloacae as a neonatal pathogen warrants the attention of clinicians as well as microbiologists. Breaches in infection control measures should be avoided to prevent such outbreaks.

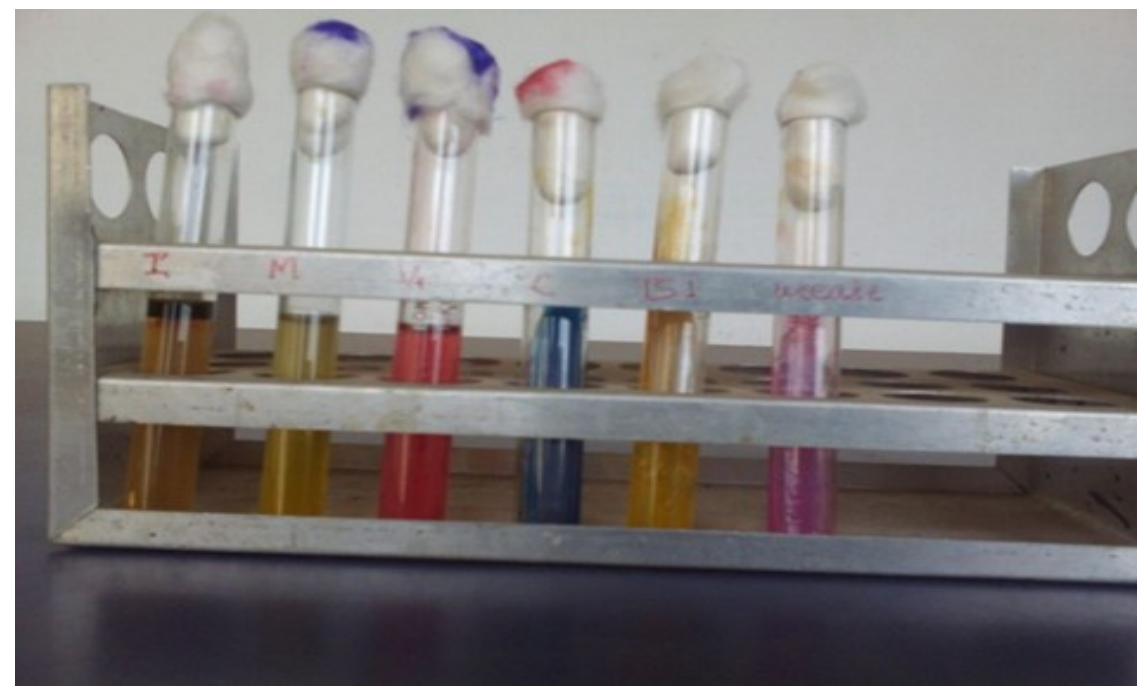

Figure -1 Biochemical properties of Enterobacter cloacae 


\section{References}

[1]. Beena Antony andRajendra Prasad BPM.An outbreak of neonatal septicaemia by Enterobacter cloacae.Asian Pacific Journal of Tropical Disease2011;227-229.

[2]. Rastogi V, Nirwan PS, Jain S andKapil A. Nosocomial outbreak of septicaemia in neonatal intensive care unit due to extended spectrum $\beta$-lactamase producing Klebsiella pneumoniae showing multiple mechanisms of drug resistance.Indian Journal of Medical Microbiology2010; 28(4): 380-384.

[3]. Petra Gastmeier, Andrea Loui, Sabine Stamm-Balderjahn, Sonja Hansen, Irina Zuschneid, DoritSohr, et al.Outbreaks in neonatal intensive care units -They are not like others.AJIC2007;172 - 176.

[4]. http://emedicine.medscape.com/article/978352-overview

[5]. Washington. W, Allen.S, Janda.W, Koneman.E, Procop.G, Schreckenberger.P, Woods.G.Koneman's Colour Atlas and Textbook of Diagnostic Microbiology.

[6]. Mearl C Mahl. New Method for Determination ofEfficacy of Healthcare Personnel Hand Wash Products. Journal of Clinical Mcrobiology Oct. 1989; 2295 - 2299.

[7]. Clinical and Laboratory standards Institiute. M100-S23. Performance Standards for Antimicrobial susceptibilityTesting TwentyThird Informational supplement; Pennsylvania. 2013

[8]. Glen MayhallC.Hospital Epidemiology \& Infection Control.Lippincott Williams \& Wilkins. $3^{\text {rd }}$ edition, $2004 ; p p 864$.

[9]. Kenneth J Ryan and C George Ray. Sherri' s Medical Microbiology; McgrawHill

[10]. Medical Publishing Division. $4^{\text {th }}$ edition, 2004; Chapter 71; pp 370.

[11]. Mahapatra A, Ghosh SK, Mishra S, Pattnaik D, Pattnaik K andMohanty SK. Enterobacter cloacae: A predominant pathogen in neonatal septicaemia. . Indian J Med Microbiol 2002;20:110- 112.

[12]. Kurlat I, Corral G andOlivera F. Infection control strategies in NICU in Argentina. Journal of Hospital Infection1998;40;149 - 154

[13]. Archibald LK,Manning ML and Bell LM. Patient density and Nurse to Patient ratio and Nosocomial infection risk in paediatric cardiac ICU. Paediatric Infect Dis 1996;15;165 - 169.

[14]. Gaynes RP, Martone WJ, Culver DH, Emori T, Horan T, Banerjee S, et al. Comparison of rates of nosocomial infections in neonatal intensive care units in the United States. Am J Med 1991;91(Suppl 3B):192 - 196.

[15]. Gastmeier P, Geffers C, Schwab F, Fitzner J, Obladen M andRudenH.Development of a surveillance system for nosocomial infections: the component for neonatal intensive care units in Germany as an example. J Hosp Infect 2004;57:126-131.

[16]. Hwang J, Choi C, Chang Y, Choe Y, Park W, Skin S, et al. The efficacy of clinical strategies to reduce nosocomial sepsis in extremely low birth weight infants. J Korean Med Sci 2005;20:177- 181.

[17]. Warren D, Zack J, Mayfield J, Chen A, Prentice D, Fraser V, et al. The effect of an education program on the incidence of central venous catheterassociated bloodstream infection in a medical ICU. Chest 2004;126:1612-18. 